\title{
Article
}

\section{RNA helicase DDX3 interacts with the capsid protein of hepatitis $E$ virus and plays an indispensable role in the viral replication}

\author{
Shaoli Lin ${ }^{1}$, Peixi Chang ${ }^{1}$, Jia He ${ }^{1}$, Etienne Coyaud ${ }^{2}$, Brian G. Pierce ${ }^{3,4}$, and Yan-Jin Zhang ${ }^{1 *}$ \\ ${ }^{1}$ Molecular Virology Laboratory, VA-MD College of Veterinary Medicine, University of \\ Maryland, College Park, MD, U.S.A. \\ ${ }^{2}$ Univ. Lille, Inserm, CHU Lille, U1192 - Protéomique Réponse Inflammatoire \\ Spectrométrie de Masse - PRISM, F-59000 Lille, France \\ ${ }^{3}$ Institute for Bioscience and Biotechnology Research, University of Maryland, Rockville, MD, \\ U.S.A. \\ ${ }^{4}$ Department of Cell Biology and Molecular Genetics, University of Maryland, College \\ Park, MD, U.S.A.
}

*Correspondence: zhangyj@umd.edu; Tel: (301) 314-6596

\begin{abstract}
DDX3 is an ATP-dependent RNA helicase involved in multiple cellular activities, including RNA metabolism and innate immunity. DDX3 is known to assist the replication of some viruses while restricting some others through direct interaction with the viral proteins. However, the role of DDX3 in the replication of the hepatitis E virus (HEV) is unknown. In this study, DDX3 is shown to interact with the HEV capsid protein and provide an indispensable role in HEV replication. The DDX3 C-terminal domain was demonstrated to interact with the capsid protein, which was previously demonstrated to inhibit the production of type I interferons. Knockdown of DDX3 compromised the capsid protein-mediated blockage of interferon induction. Notably, DDX3 silencing led to a significant reduction in HEV replication. Also, the ATPase activity of DDX3 is required for the HEV replication as an ATPase-null mutant DDX3 failed to rescue the viral replication in the DDX3-silenced cells. These results demonstrate a pro-viral role of DDX3 in HEV replication, providing further insights into the virus-cell interactions.
\end{abstract}

Key words: Hepatitis E virus (HEV), interferon (IFN), the capsid protein, DDX3, RNA helicase 


\section{INTRODUCTION}

Helicases are divided into six superfamilies on the basis of sequence homology in conserved motifs [1]. The DEAD (Asp-Glu-Ala-Asp)-box RNA helicases are members of the superfamily 2 and are ubiquitously expressed in all eukaryotes and most prokaryotes [2,3]. DEADbox helicase 3, X-linked (DDX3X), is involved in almost all aspects of RNA metabolism from RNA transcription, splicing, transport, translation to decay. DDX3X is also involved in innate immunity, viral infection, and tumorigenesis [3-6]. DDX3X is located on the $\mathrm{X}$ chromosome and is present in all human tissues. The deletion of DDX3X in male mice is embryonic lethal [7]. DDX3X has a paralog, DDX3Y, which is located on the Y chromosome and is closely related to male fertility [8]. It is observed that DDX3Y is frequently lost in infertile patients [9].

DDX3X (hereinafter referred to as DDX3) consists of two RecA-like domains, domain 1 (aa166-407) and 2 (aa414-576). Mutation of the lysine 230 residue within the N-terminal ATPase motif of DDX3 results in the loss of ATPase activity. Mutation of serine 382 residue within the helicase motif abolishes the RNA unwinding activity of DDX3 [10]. DDX3 couples with mitochondrial antiviral-signaling protein (MAVS) to promote the transcription of interferons (IFNs). Knockdown of DDX3 reduces IFN production [11]. Previous studies have demonstrated that DDX3 is required for viral replication for some viruses [3,12]. During HCV replication, DDX3 is recruited to the lipid droplets, where HCV virions assemble. Knockdown of DDX3 in hepatoma cells significantly inhibits HCV replication [13]. DDX3 promotes arenavirus replication by enhancing its RNA synthesis. DDX3 with mutations of either the ATPase motif (K230E) or the helicase motif (S382A) fails to promote the arenavirus RNA synthesis [14]. In contrast to the findings above, DDX3 can exert an inhibitory role for some other viruses. DDX3 interacts with influenza virus NS1 and NP via its C-terminal domain and associates with stress granules to inhibit viral infection [15]. Silencing DDX3 attenuates the formation of stress granules and also enhances viral replication. DDX3 is also shown to inhibit HBV replication by interacting with polymerase and incorporating itself into the nucleocapsid. The ATPase activity of DDX3 is essential for viral inhibition [16].

Hepatitis E virus (HEV) is a single-stranded positive-sense RNA virus that is one of the most common causes of acute hepatitis. HEV infection can cause fulminant hepatic failure, leading to a high case-fatality rate among infected pregnant women in developing countries [1719]. Acute HEV infection also causes substantial morbidity in pregnant women in developed countries [20]. HEV strains are heterogenic, and at least four genotypes can infect humans: genotypes 1 and 2 are restricted to humans, whereas genotypes 3 and 4 are zoonotic with an expanded host range. The HEV genome contains three open reading frames, among which ORF1 encodes the non-structural polyprotein for viral RNA replication, ORF2 for the capsid protein, and ORF3 for a multifunctional protein. HEV is understudied, and its mechanistic insights of $\mathrm{HEV}$-cell interactions remain largely unknown.

The capsid protein is demonstrated to inhibit the IFN induction via blocking phosphorylation of interferon regulatory factor 3 (IRF3) [21]. The objective of this study was to determine if other factors are involved in the inhibition. Here, DDX3 was found to associate with the HEV capsid protein. Knockdown of DDX3 compromises the capsid protein-mediated IFN inhibition. Notably, the DDX3 knockdown inhibits the HEV replication, suggesting DDX3 plays an indispensable role in the viral infection. Mutagenesis analysis shows that the ATPase activity 
of DDX3 is required for HEV replication. These results reveal the critical role of DDX3 in HEV proliferation and provide insights into the virus-cell interactions.

\section{MATERIALS AND METHODS}

2.1. Cells and viruses. Huh7.5.1 [22], GP2-293 (Clotech Laboratories, Inc., Mountain View, CA), HEK293T (ATCC ${ }^{\circledR}$ CRL-3216 ${ }^{\mathrm{TM}}$ ), and HepG2/C3A (ATCC® CRL-10741 ${ }^{\mathrm{TM}}$ ) cells were maintained in Dulbecco's modified Eagle's medium (DMEM) supplemented with 10\% fetal bovine serum (FBS). In vitro transcription was performed from p6-luc, an HEV replicon that contains cDNA of the genomic RNA of Kernow-C1, a genotype 3 strain, with an insert encoding Gaussia luciferase replacing the 5 ' portion of ORF2 [23]. The luciferase with a signal peptide is secreted out of the cells after synthesis. The Kernow-C1 strain p6 was used to infect HepG2/C3A cells at a multiplicity of infection (MOI) of 1 . The infected HepG2/C3A cells were passaged five times to produce stably infected cells.

2.2. Plasmids. The ORF2 from HEV Kernow-C1 strain (GenBank Accession Number: JQ679013) was cloned to the Myc-BioID2-MCS vector (a gift from Kyle Roux, Addgene plasmid \# 74223; http://n2t.net/addgene:74223 ; RRID:Addgene_74223) [24] for the expression of a fusion protein. DDX3 ORF clone (NM_001356) was purchased from OriGene Technologies (Rockville, MD) and was amplified by PCR for subcloning to the pCAGEN vector with a FLAG tag at the N-terminus, as described previously [21]. The DDX3 mutants (K230E and S382L) were generated by overlapping PCR and ligated to the PCAGEN-HA vector [21]. DDX3 D1 and D2 truncations were ligated to PCAGEN with an HA tag at the C-terminus. The oligos for shRNA against DDX3 (Table 1) were cloned to pSIREN-RetroQ-ZsGreen vector as instructed (Clotech). Primers used in this study are listed in Table 1. All plasmids constructed in-house were subjected to verification by Sanger DNA sequencing.

Table 1. Primers used in this study

\begin{tabular}{lll}
\hline Primer $^{\mathbf{a}}$ & Sequence (5' to 3') $^{\mathbf{b}}$ & Target \\
\hline KH2F4 & GCTCGAGTGCCCTAGGGTTGTTCTGCTGCTGTTC & Kernow ORF2 \\
KH2R4 & ACGTCTCGAATTCTTAAGACTCCCGGGTTTTGCCTACCTCCG & Kernow ORF2 \\
DDX3-F1 & CGAATTCAGTCATGTGGCAGTGGAAAAT & DDX3 \\
DDX3-R1 & ACTCGAGTCAGTTACCCCACCAGTCAAC & DDX3 \\
DDX3-D1-F1 & TGAATTCGCCACCATGAGTCATGTGGCAGTGGA & DDX3 D1 \\
DDX3-D1-R1 & GCTCGAGTTCAGAGGTAGAGCCAACTCT & DDX3 D1 \\
DDX3-D2-F2 & TGAATTCGCCACCATGGCTGTAGGAAGAGTTGGCTC & DDX3 D2 \\
DDX3-D2-R2 & GCTCGAGGTTACCCCACCAGTCAACC & DDX3 D2 \\
shDDX3-F1 & GATCCGGAGTGATTACGATGGCATTGTTCAAGAGACAATGC & DDX3 \\
shDDX3-R1 & CATCGTAATCACTCCTTTTTG & \\
& CAATCAAAAAAGGAGTGATTACGATGGCATTGTCTCTTGAAATCAATCACTCCG & DDX3
\end{tabular}




$\begin{array}{lll}\text { DDX3-K230E-F1 } & \begin{array}{l}\text { TGTGCCCAAACAGGGTCTGGAGAGACTGCAGCATTTCTGTTG } \\ \text { CCC }\end{array} & \text { DDX3 K230E } \\ \text { DDX3-K230E-R1 } & \begin{array}{l}\text { GGGCAACAGAAATGCTGCAGTCTCTCCAGACCCTGTTTGGG } \\ \text { CACA }\end{array} & \text { DDX3 K230E } \\ \text { DDX3-S382L-F1 } & \text { TTCCTTAGGAAAAGTAGCCAAAAACATCATAGTGTGGCG } & \text { DDX3 S382L } \\ \text { DDX3-S382L-R1 } & \text { CGCCACACTATGATGTTTAGTGCTACTTTTCCTAAGGAA } & \text { DDX3 S382L }\end{array}$
a. F: forward primer, R: reverse primer. ORF2 sequence is from HEV Kernow-C1 P6 (GenBank accession\# JQ679013).
b. The italicized alphabets indicate restriction enzyme cleavage sites for cloning.

2.3. Transfection. Transfection of cells with plasmid DNA was done with jetOPTIMUS $®$ DNA transfection Reagent (Polyplus transfection, New York, NY) according to the instructions of the manufacturer. Low molecular weight Poly(I:C), a synthetic analog of double-stranded RNA (dsRNA) (Invivogen, San Diego, CA), was used to induce interferon production. GP2-293 cells were transfected with poly(I:C) at a concentration of $1 \mu \mathrm{g} / \mathrm{ml}$ for overnight before harvested for further analysis.

2.4. DDX3 knockdown. GP2-293 cells were co-transfected with pSIREN-RetroQZsGreen-shDDX3 and pVSV-G plasmids at a ratio of 1:1. At 48 hours later, the culture supernatant was harvested and subjected to centrifugation at $10,000 \mathrm{xg}$ for $2 \mathrm{~min}$ to eliminate the cell debris. The cleared supernatant was added to Huh7.5.1 cells, followed by incubation for 3 days.

2.5. BioID assay. HEK293T cells in a 10-cm dish were transfected with Myc-BioID2pORF2 or the empty vector Myc-BioID2-MCS. At 24h later, the cells were treated with biotin at a final concentration of $5 \mathrm{mM}$. The cells were harvested with lysis buffer $(50 \mathrm{mM}$ Tris $\mathrm{HCl}, \mathrm{pH} 7.4$, $500 \mathrm{mM} \mathrm{NaCl}, 0.2 \% \mathrm{SDS}$ (W/V), with the addition of the following before use: 1x protease inhibitor cocktail (Sigma-Aldrich, St. Louis, MO) and $1 \mathrm{mM}$ DTT) at $24 \mathrm{~h}$ after biotin treatment. The cell lysate was subjected to dialysis against PBS pH7.2 to eliminate the free biotin overnight. Then the cell lysate was incubated with streptavidin-conjugated magnetic beads at $4^{\circ} \mathrm{C}$ overnight. The beads were rinsed for four times with the washing buffer, resuspended with $200 \mu \mathrm{l}$ of PBS, incubated with biotin at a final concentration of $5 \mathrm{mM}$ for $5 \mathrm{~min}$ before addition of $2 \mathrm{X}$ Laemmli sample buffer, and heated at $100^{\circ} \mathrm{C}$ for $10 \mathrm{~min}$ to elute the biotinylated proteins [25]. The elute was subjected to sulfate-polyacrylamide gel electrophoresis (SDS-PAGE) in-house and mass spectrometry analysis by Taplin Mass Spectrometry Facility, Harvard Medical School (Boston, MA). Briefly, gel pieces were subjected to a in-gel trypsin digestion procedure [26]. Peptides were extracted and high performance liquid chromatography was conducted to elute the sample through a C18 column. The samples were run in a LTQ Orbitrap Velos Pro ion-trap mass spectrometer (Thermo Fisher Scientific, Waltham, MA). Peptide sequences (and hence protein identity) were determined by Sequest software analysis (Thermo Fisher Scientific, Waltham, MA) [27]. All databases include a reversed version of all the sequences and the data was filtered to between a one and two percent peptide false discovery rate. Total peptide counts were compared against 12 BioID control runs ( HEK293) extracted from the CrapOME depository (http://www.crapome.org/; [28]). Proximal interactors hits were defined based on the following thresholds: detected with at 
least three unique peptides and a fold change greater than five times the average control runs (12 CrapOME + a study control) for each given ID (Supplemental Table 1).

2.6. Electroporation. The Huh7.5.1 cells in a 6-well plate were detached with trypsin, rinsed with Opti-MEM twice, and resuspended in $200 \mu$ Opti-MEM. HEV RNA was added to the tubes at $4 \mu \mathrm{g}$ each and mixed gently. The mixture of cell suspension and RNA was then transferred to a 0.4-cm cuvette for pulsation in Gene Pulser Xcell electroporation (Bio-Rad) under the condition of $600 \mathrm{~V}, 1$ pulse, and 0 intervals. The cells were then plated for culture.

2.7. Luciferase reporter assay and cell viability assay. After the electroporation, samples of culture supernatant at $100 \mu \mathrm{l}$ were taken from each well once every $24 \mathrm{~h}$ and frozen at $-80^{\circ} \mathrm{C}$. Same amount of fresh medium was then added to the culture for further incubation. Supernatant samples from the entire experiment were thawed together and assayed for luciferase activity using the Renilla luciferase assay system (Promega) according to the manufacturer's protocol. Briefly, supernatant samples were added to a 96-well black, flat-bottom microplate (Corning), followed by the addition of the same amount Renilla luciferase assay substrate and the detection of luminescence using a PerkinElmer 1420 Multilabel Counter. Three replicates were tested for each group. The CellTiter-Glo Luminescent Cell Viability Assay kit (Promega) was used to determine cell viability by following the manufacturer's instructions.

2.8. Co-Immunoprecipitation (Co-IP) and Western blotting. Co-IP was conducted with the antibodies against FLAG (Sigma-Aldrich) and HEV capsid protein (homemade) as described [29]. Protein A/G Magnetic Beads for IP (Bimake, Houston, TX) were used following the manufacturer's instructions. The IP samples were subjected to Western blot analysis with the antibodies against Myc, HA, and the capsid protein. SDS-PAGE and Western blotting were done as previously described [21]. Antibodies against DDX3 (Proteintech Group, Inc, Rosemont, IL), HA (Thermo Fisher Scientific, Waltham, MA), Myc (Thermo Fisher), FLAG (Sigma-Aldrich), GAPDH (Santa Cruz Biotechnology, Inc., Dallas, TX), and $\beta$-tubulin (Sigma-Aldrich) were used in the immunoblotting. Collection of the chemiluminescence signal was done with the Quantity One Program, version 4.6 (Bio-Rad Laboratories, Hercules, CA) and a ChemiDoc XRS imaging system (Bio-Rad).

2.9. Reverse transcription and real-time quantitative PCR (RT-qPCR). Total RNA was isolated from GP2-293 cells with TRIzol reagent (Thermo Fisher). Reverse transcription was done with Moloney murine leukemia virus reverse transcriptase (Thermo Fisher), oligo (dT), and a random 15-mer oligo. Quantitative PCR of IFN- $\beta$ and RPL32 (ribosomal protein L32, as an internal control) transcripts was performed using SYBR Green Supermix (Thermo Fisher) as described [30].

2.10. Computational analysis of protein-protein interaction. The structure of the HEV Kernow-C1 ORF2 (capsid) protein in dimeric form was generated using the homology modeling program Modeller, with a previously determined structure of the HEV capsid [31] (Protein Data Bank [32] code 2ZTN) used as a template. This structure, and the previously determined structure of human DDX3 [33] (Protein Data Bank code 5E7I) were input to the ZDOCK server (http://zdock.umassmed.edu/) [34] to generate a model of the DDX3-capsid complex, with default docking parameters used for server input. The top-ranked ZDOCK server model (complex 1) is shown in the Results.

2.11. Protein network analysis. The subcellular distribution of the biotinylated proteins from the mass spectrometry analysis was performed by ToppFun server 
(https://toppgene.cchmc.org/enrichment.jsp; Supplemental Table 1) and Cytoscape version 3.6.1.

2.12. Statistical analysis. Differences in indicators between treated samples, such as the Gaussia luciferase level between DDX3-silenced cells and control cells, were assessed by the Student $t$-test. A two-tailed $\mathrm{P}$ value of less than 0.05 was considered significant.

\section{RESULTS}

\subsection{BioID and mass spectrometry analysis}

Our earlier study showed that the capsid protein inhibits type I interferon production via its arginine-rich motif (ARM) [35]. We hypothesized that the capsid protein might influence IFN production through additional cellular factors. To assess this hypothesis, we performed a pilot BioID assay to get insight into which host proteins are present in close proximity to the capsid protein. HEK293T cells were transfected with Myc-BioID2-pORF2 or Myc-BioID2-MCS empty vector, and biotin was added for the biotinylation of adjacent proteins. The biotinylation of cellular proteins was verified by Western blotting. Comparing the ORF2 BioID data against our control and a set of CrapOME controls, we identified 145 potential proximal interactors (Supplemental Table 1; see Materials and Methods section). These hits were analyzed in ToppFun to identify enriched GO Cellular Component categories (details in Supplemental Table 1). This analysis showed that the capsid protein was associated with the mitochondrion proteins (GO:0005739: 26/145; Figure 1A), suggesting that the capsid protein may interfere with some mitochondrial processes. Additionally, a great portion of the identified proteins was predicted to localize to ribonucleoprotein granules (GO:0035770: 24/145), supporting a role of the capsid protein in viral genome binding or post-transcriptional regulation of cellular RNA. GO Biological Process analysis revealed that ORF2 proximal interactors were highly enriched in proteins assigned to e.g. RNA metabolism (GO:0016071: 28/145), regulation of translation (GO:0006417: 20/145) and proteolysis (GO:0006508: 29/145)y (Figure 1B).

A

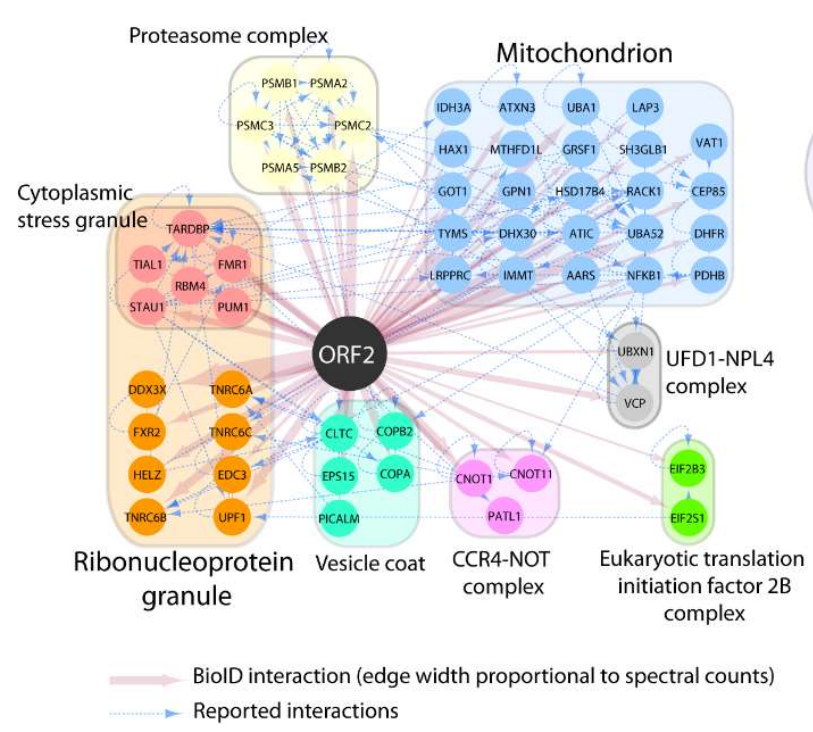

B

Regulation of translation RNA catabolic process

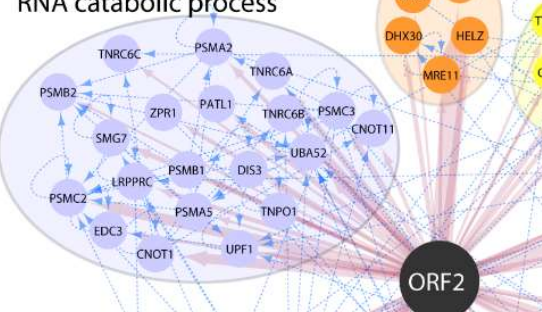

Identical protein binding

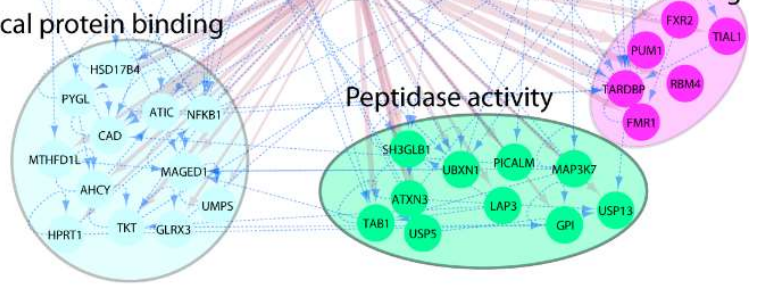

Figure 1. pORF2-proximal proteins enrichment. Selection of pORF2 interactors indicated by color-coded nodes and grouped according to their intracellular localization (GO CC; A) 
and functions (GO BP; B). Selected hits and corresponding relevant categories as indicated. BioID interactions are depicted in red and reported interactions between hits (collected from http://iid.ophid.utoronto.ca/; using experimental evidence as a threshold; sources detailed in Supplemental Table 2) are shown by dashed blue edges.

\subsection{The capsid protein interacts with DDX3 independent of RNA association}

The BioID/MS data analysis shows the top candidate with the highest sum intensity and number of the peptides matched to DDX3, suggesting a strong interaction with the capsid protein. To test the hypothesis, we further tested the interaction between DDX3 and the capsid protein by Co-IP. HEK293T cells were co-transfected with FLAG-DDX3 and Myc-BioID2pORF2. Co-IP with FLAG antibody was conducted to precipitate DDX3, followed by Western blotting with Myc antibody for detection of the Myc-tagged capsid protein. The result showed that the capsid protein was present in the precipitates of DDX3, thus indicating a physical interaction (Figure 2A). Since both DDX3 and the capsid protein are RNA-associated proteins, we wondered whether the interaction between the two molecules was mediated by RNA. HEV RNA was transfected to the cells as the capsid protein is known to interact with the viral RNA, followed by Co-IP in the presence of RNase A. The result showed that the RNase treatment did not affect the co-precipitation of DDX3 and the capsid protein (Figure 2B), indicating the interaction is independent of their RNA binding function.

A

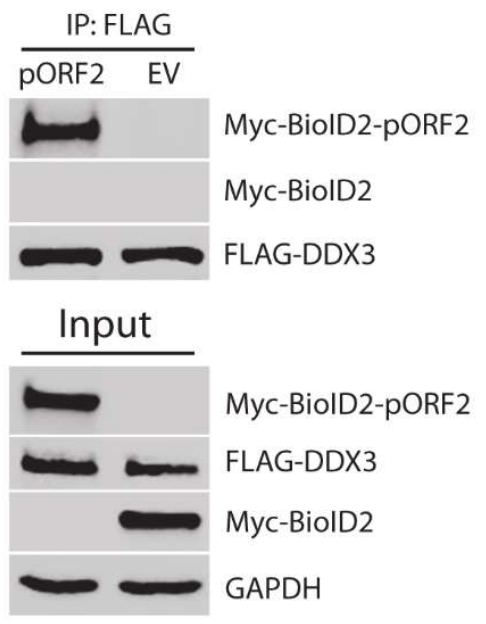

B
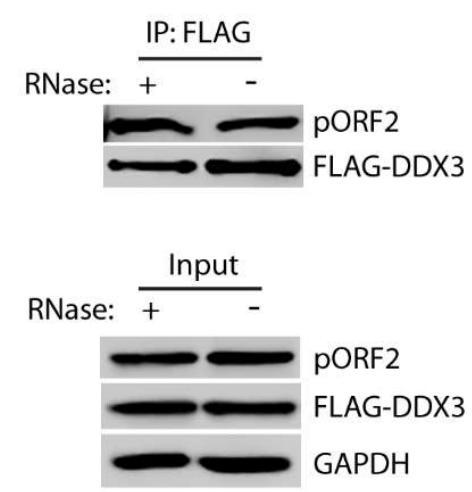

Figure 2. DDX3 co-precipitates the capsid protein, independent of RNA association. A. Co-IP of DDX3 precipitates the capsid protein. HEK293T cells were co-transfected with FLAGDDX3 and Myc-BioID2-pORF2, and harvested for Co-IP with the FLAG antibody at 36 hosttransfection (hpt). The IP precipitate and input were subjected to Western blotting (WB). BioID2 empty vector was used as a negative control. B. The co-precipitation of DDX3 and the capsid protein is independent of RNA. HEK293T cells were co-transfected with FLAG-DDX3 and Myc-BioID2-pORF2. At $24 \mathrm{hpt}$, the cells were further transfected with RNA from in vitro transcribed Kernow p6 replicon as a control. The cells were harvested $24 \mathrm{~h}$ post RNA transfection. The cell lysate was treated with RNase A at $333 \mu \mathrm{g} / \mathrm{ml}$ for $20 \mathrm{~min}$ at room temperature, followed by Co-IP and WB. 


\subsection{HEV capsid protein interacts with the C-terminal region of DDX3}

In order to map the domains that are responsible for the capsid protein and DDX3 interaction, modeling of the complex structure was performed with the ZDOCK server [34]. The top-ranked model shows a possible interaction between the C-terminal domain of DDX3 and the C-terminal domain of the capsid protein dimer (Figure 3A), which corresponds to the "P" domain that protrudes from the surface of the full capsid assembly [31]. Two DDX3 truncations were constructed: N-terminal D1 and C-terminal D2 (Figure 3B) to test the prediction. The DDX3 truncations and ORF2 plasmids were co-transfected into HEK293T cells. Co-IP with the antibody against the capsid protein showed that DDX3-D2 but not DDX3-D1 was present in the precipitates (Figure 3C), which indicates that the $\mathrm{C}$-terminal domain of DDX3 interacts with the capsid protein.

A

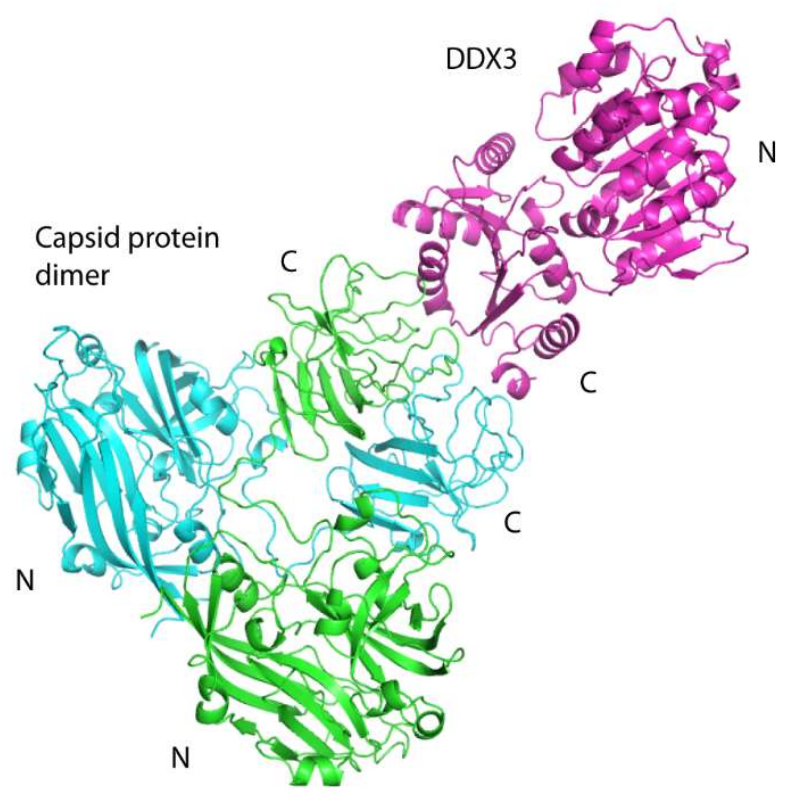

B

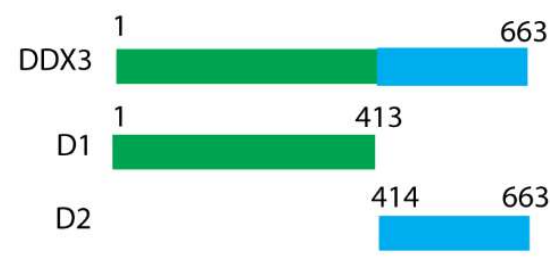

C

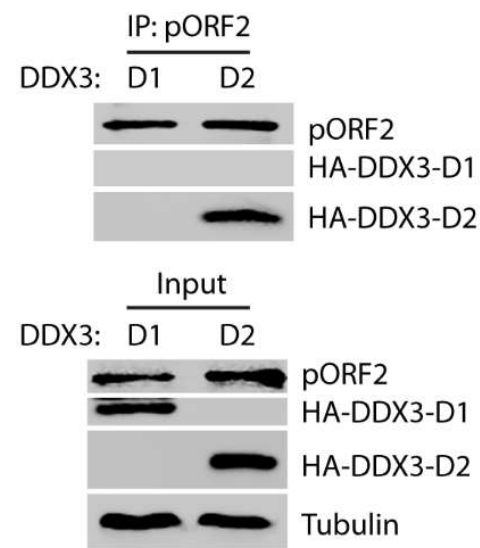

Figure 3. The C-terminal domain of DDX3 interacts with the capsid protein.

A. A model of the interaction between DDX3 and the capsid protein. The capsid protein is in homodimeric form and chains are colored green and cyan, and DDX3 is colored magenta. "N" stands for $\mathrm{N}$-terminus and " $\mathrm{C}$ " for C-terminus; these are labeled for each protein. B. Schematic illustration of the two DDX3 domains and the truncations. The numbers above the lines indicate amino acid residues of DDX3. C. The capsid protein co-precipitates the C-terminal domain of DDX3. HEK293T cells were transfected with plasmids of DDX3-D1, D2, and pORF2. Co-IP with the antibody against pORF2 was conducted, followed by Western blotting with an antibody against HA for DDX3. Cell lysate input was included in the Western blotting as a control.

\subsection{Knockdown of DDX3 impedes the IFN induction and the capsid protein-mediated inhibition}

Since DDX3 enhances MAVS-mediated IFN signaling [36] and interacts with the capsid protein, we wondered whether this association is needed for the capsid protein-mediated inhibition of IFN signaling. In order to test this, RNAi-mediated silencing of DDX3 in GP2-293 cells was conducted. Compared to the control shRNA, the shRNA against DDX3 (shDDX3) 
significantly reduced the DDX3 level (Figure 4A). The shRNA treatment had minimal effect on cell growth (Figure 4B). The cells were then transfected with ORF2 plasmid and treated with poly(I:C). The expression of the IFN- $\beta$ was then determined by RT-qPCR. The result showed that the knockdown of DDX3 attenuated the IFN induction by over $50 \%$, and the capsid protein's inhibition on IFN was compromised, indicating that DDX3 contributes to the capsid proteinmediated inhibition of IFN signaling (Figure 4C).

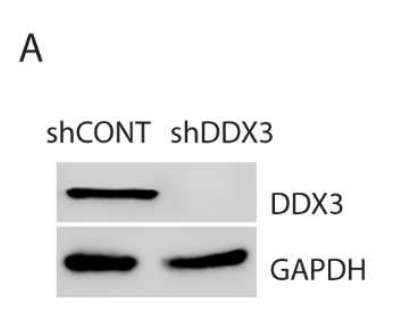

B

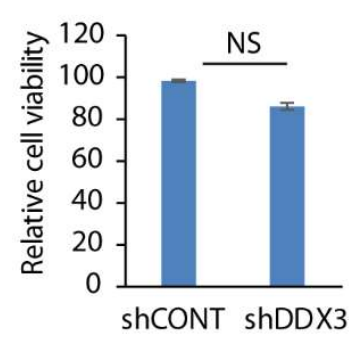

C

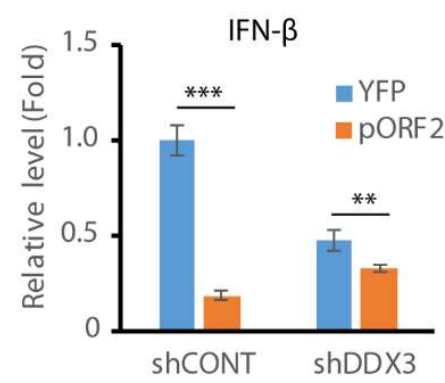

Figure 4. Knockdown of DDX3 compromises the capsid protein's inhibition of IFN. A. Knockdown of DDX3 in GP2-293 cells. The cells were transfected with pSIREN-RetroQZsGreen-shDDX3 and pVSV-G plasmids for 3 days and were then harvested for WB. B. DDX3 silencing has minimal effect on GP2-293 cell viability. GP2-293 cells were co-transfected with pSIREN-RetroQ-ZsGreen-shDDX3 and pVSV-G plasmids for 3 days and were then harvested for cell viability assay. NS: no significant difference. C. DDX3 silencing leads to a significant reduction of poly(I:C)-induced interferon expression. DDX3-silenced GP2-293 cells were transfected with the capsid protein or YFP plasmid for 24 hours and then transfected with Poly(I:C) overnight. The cells were harvested for RNA extraction and RT-qPCR for IFN- $\beta$ expression. Transcript of house-keeping gene RPL32 was also determined for normalization. Relative levels in comparison with the YFP and shRNA control are shown. *** denotes $P<0.001$ and $* *$ for $P<$ 0.01 .

\subsection{DDX3 knockdown reduces the replication of HEV.}

In order to verify the role of DDX3 during HEV replication, Huh7.5.1 cells were transduced with a retrovirus containing shDDX3 or a control shRNA. The shDDX3-based gene silencing led to the knockdown of DDX3 compared to the control shRNA (Figure 5A). Cell viability assay was done when the cells were treated with shDDX3 for 3 days. The result showed that the DDX3 knockdown had minimal effect on cell growth in comparison with the control shRNA (Figure 5B). The DDX3-silenced Huh7.5.1 cells were electroporated with the RNA of HEV replicon p6/luc that encodes a secretory Guassia luciferase, an indicator of viral replication. The cell culture supernatant samples were collected every day for 8 consecutive days after the transfection. Luciferase assay result showed that HEV replication was significantly impaired by the knockdown of DDX3, compared with the cells of control shRNA (Figure 5C). The luciferase activity increased from day 3 and peaked at day 5 in the control cells, whereas the increase was quite limited in the DDX3-silenced cells.

In order to exclude the possibility of an off-target effect of the shRNA, the DDX3-silenced Huh7.5.1 cells were transfected with DDX3 plasmid to trans-compensate the expression. WB result demonstrated the ectopic expression of DDX3 (Figure 5D). The DDX3-silenced Huh7.5.1 cells with DDX3 trans-compensation were transfected with the RNA of HEV replicon p6/luc. 
Luciferase activity assay of the culture supernatant sample of day 5 after electroporation showed that the DDX3 reconstitution led to the restoration of most HEV replication, whereas the empty vector control failed to do so (Figure 5E).

The results above demonstrate the essential role of DDX3 in HEV replication, shown by an HEV replicon with luciferase. To further verify the role of DDX3 in the replication of wildtype HEV, we conducted DDX3 knockdown in HepG2/C3A cells stably infected with HEV Kernow-C1 strain. WB result showed the DDX3 deficiency in the cells three days posttransduction with retrovirus encoding shDDX3 (Figure 5F). RT-qPCR result showed HEV RNA level in the DDX3-silenced cells was only $37 \%$ of that in the control cells (Figure 5G). These results demonstrated that DDX3 is indispensable for HEV replication.

A

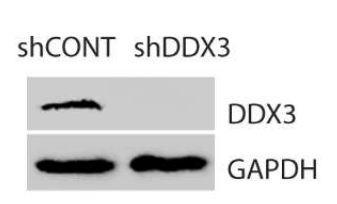

B

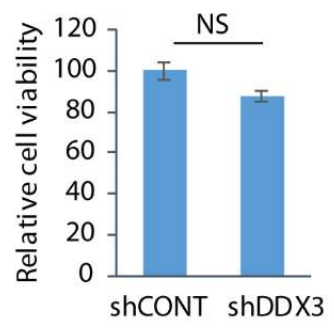

C

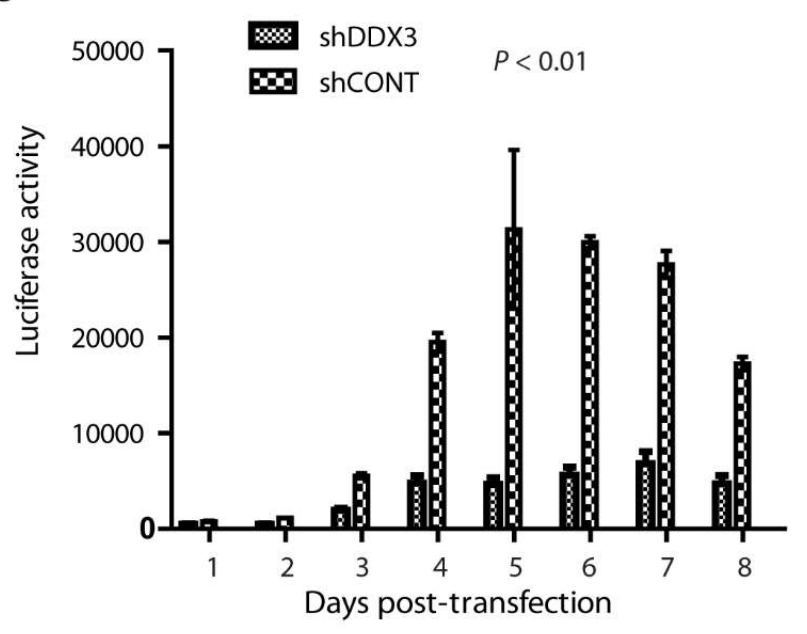

D

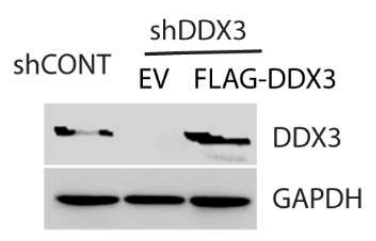

$\mathrm{E}$

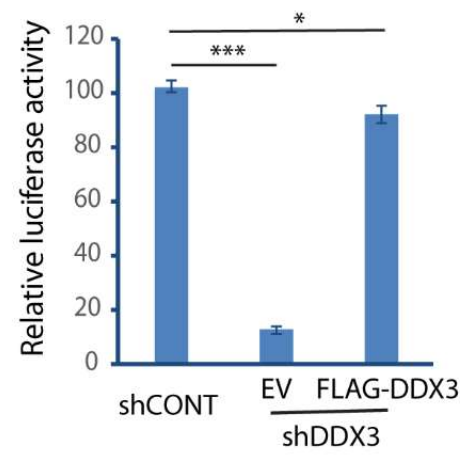

$\mathrm{F}$

shCONT ShDDX3

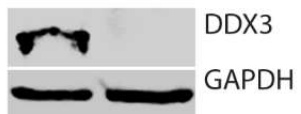

G

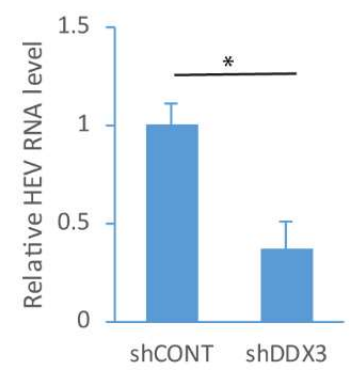

Figure 5. Knockdown of DDX3 results in a significant reduction of HEV replication. A. Western blot detection of DDX3 in Huh7.5.1 cells after the DDX3 knockdown. The cells were transduced with a retrovirus containing DDX3-shRNA (shDDX3) or control shRNA (shCONT) for 3 days and were harvested for WB detection of DDX3. B. Knockdown of DDX3 
has minimal effect on the cell growth. The Huh7.5.1 cells were transduced with a retrovirus containing shDDX3 for 3 days before the cell viability was tested. C. DDX3 knockdown reduces luciferase yield from HEV replicon. Huh7.5.1 cells with or without DDX3 silencing were electroporated with RNA of p6/luc replicon. The daily culture supernatant samples were assayed for luciferase activity. D. WB of DDX3 trans-compensation in DDX3-silenced Huh7.5.1 cells. The cells were transduced with retrovirus encoding shDDX3 for 2 days and then transfected with FLAG-DDX3 or EV (empty vector) for another 2 days. E. Ectopic expression of DDX3 restores most HEV replication. The Huh7.5.1 cells with DDX3 reconstitution were electroporated with the RNA of HEV replicon p6/luc. The luciferase activity in the culture supernatant samples was tested 5 days later. $* * *$ denotes $P<0.001$ and $* *$ for $P<0.01$. F. Knockdown of DDX3X in HepG2/C3A cells stably infected with HEV Kernow-C1 strain. The cells were transduced with retrovirus encoding shDDX3 or control. G. HEV RNA level in DDX3-silenced HepG2/C3A cells. The Kernow-infected cells were harvested for RNA isolation three days of post-shDDX3 treatment. Transcript of RPL32 was also determined for normalization. Relative levels in comparison with shRNA control are shown. * denotes $P<0.05$.

\subsection{The ATPase activity of DDX3 is required for HEV replication}

Since both DDX3 ATPase and helicase activities are frequently involved in the protein functions, we wondered whether both the ATPase and helicase activities are required for the HEV replication. We constructed the ATPase mutants (K230E) and helicase mutants (S382L) (Figure 6A) and transfected them to DDX3-silenced Huh7.5.1 cells. Wild type DDX3 plasmid and a YFP vector were included as controls. WB result showed the expression of wild type and mutant DDX3 in the cells was at a similar level (Figure 6B). The DDX3-silenced Huh7.5.1 cells with the ectopic expression of wild-type or mutant DDX3 were electroporated with RNA of HEV replicon p6/luc. After the electroporation, culture supernatant samples were collected on day 5 for luciferase assay. The result showed that, compared to wild-type DDX3, the ATPase (K230E) mutant failed to restore the luciferase yield from the HEV replicon, whereas the helicase mutant (S382L) restored the yield (Figure 6C). The data indicate that the ATPase but not the helicase activity of DDX3 is indispensable for HEV replication.
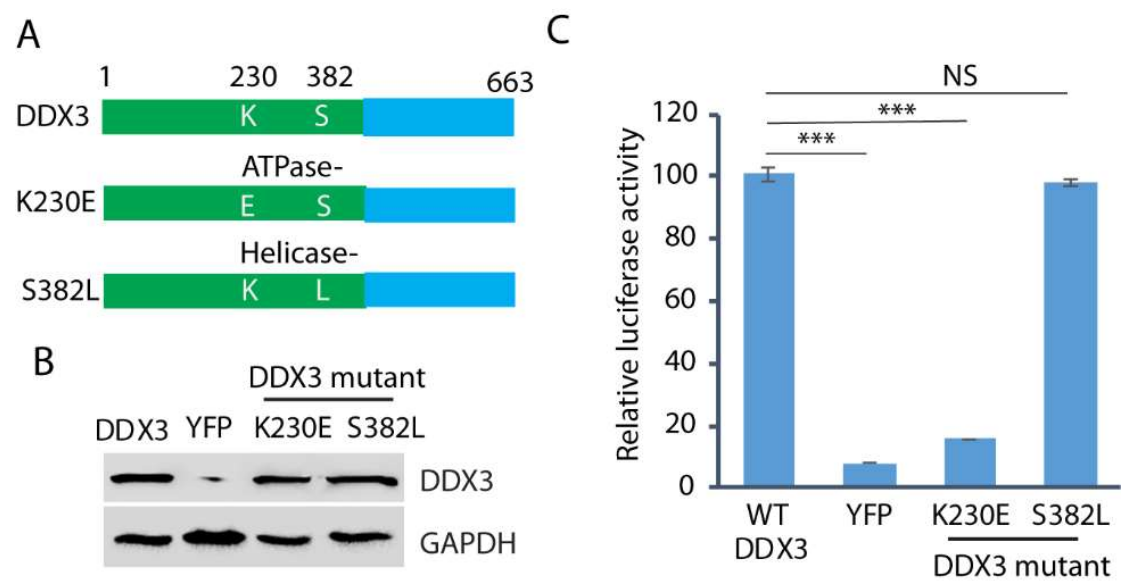

Figure 6. The ATPase activity of DDX3 is indispensable for HEV replication.

A. Schematic illustration of DDX3 and its mutants. The numbers above the lines indicate amino acid residues of DDX3. B. WB detection of the protein expression of the DDX3 and mutants in DDX3-silenced Huh7.5.1 cells. The Huh7.5.1 cells were transduced with retrovirus encoding 
shDDX3 for 2 days and then transfected with DDX3, YFP vector, or mutant DDX3 (K230E and S382L) plasmids for another 2 days. C. Restoration of HEV replication in cells transcompensated with wild-type and helicase-mutant but not ATPase-mutant DDX3. Two days after the reconstitution with wild-type or mutant DDX3, the Huh7.5.1 cells were electroporated with RNA of HEV replicon p6/luc. Luciferase activity in the culture supernatant samples was determined on day 5 . Relative luciferase activity is shown. NS: no significant difference; $* * *: P$ $<0.001$.

\section{Discussion}

The HEV capsid protein is the major component of HEV virions, but its interaction with host proteins is less understood. The BioID/MS pilot analysis in this study identifies many putative interacting cellular partners, which link the viral protein to different subcellular compartments and a broad array of putative accessory functions, including RNA metabolism and protease activity. Consistent with our earlier findings that the capsid protein inhibits MAVSactivated IFN induction, many putative interacting proteins localize to the mitochondrion, suggesting that the capsid protein may also affect the other mitochondrion-associated cellular activities. Many other interacting candidates are localized to the ribonucleoprotein granules with RNA-processing activities. Among the top candidates, DDX3 was validated to interact with the capsid protein by Co-IP. Notably, DDX3 was shown to be indispensable for HEV replication.

DDX3 is a member of the helicase family involved in cellular RNA regulation and is often involved in viral replication through either viral RNA transportation or translation [10]. In this study, the knockdown of DDX3 attenuates the HEV replication. Given the putative functions of DDX3 in RNA metabolism, we postulated that its ATPase or helicase function might be needed for viral replication. Through the mutagenesis study, we showed that the mutant DDX3 with K230E mutation failed to rescue the HEV replication in DDX3-depleted Huh7.5.1 cells, indicating the involvement of the ATPase activity of DDX3 in the viral replication. The involvement of DDX3 in the viral replication may be mediated by a direct association of DDX3 and viral genome RNA independent of its interaction with the capsid protein since the ORF2-null HEV replicon p6/luc was used. The expression of the luciferase in the replicon is from subgenomic RNA, which is synthesized by HEV RdRp [37,38]. DDX3 is known to involve in translational initiation and facilitate the newly assembled 80S ribosome [39,40]. The DDX3 knockdown possibility affects the translation of HEV RNA, leading to a low yield of RdRp and, consequently, little subgenomic RNA and luciferase production.

DDX3 synergizes with MAVS and TBK1 to enhance the production of IFNs [4,11]. Many viruses counteract this function. For instance, HCV's core protein abolishes the IFN enhancement through interaction with MAVS [36]. In this study, the knockdown of DDX3 attenuated the production of IFN and compromised the capsid protein-induced IFN inhibition, indicating the capsid protein's negative regulation on the DDX3 function. DDX3 was found to interact with the capsid protein. The interaction between these two proteins is independent of RNA, although both of them are known to bind RNA. The HEV capsid protein inhibits the production of both type I and type III IFNs by interacting with the MAVS-TBK1-IRF3 complex to block the phosphorylation of IRF3 [21]. The interaction of DDX3 and the capsid protein potentially affects the function of the MAVS-TBK1-IRF3 complex, leading to attenuation of IFN induction. 
DDX3 consists of two RecA-like domains. The C-terminal domain of DDX3 was found to be responsible for the interaction with the capsid protein. DDX3 domain 2 needs to rotate approximately $180^{\circ}$ compared to domain 1 to obtain the closed conformation that is essential for RNA binding. The binding of the mononucleotide AMP helps to stabilize the DDX3 structure [41]. DDX3 contains nine highly conserved motifs of a DEAD-box family RNA helicase: motifs Q, I, II, Ia, Ib, and III are located in domain 1, and motifs IV, V, and VI are found in domain 2. Structural, biochemical, and mutational analyses demonstrate that the different motifs are critical in various processes, such as nucleotide-binding (Q, I, and II), RNA binding (Ia, Ib, IV, and V), and ATP hydrolysis (III and possibly VI) [2]. DDX3 interacts directly with dsRNA through the N-terminal D1 domain. The contact residues are G302 and G325, and the engagement of RNA to DDX3 helps to trigger the ATPase activity of DDX3 [42]. After binding of the DDX3:dsRNA complex with ATP, DDX3 unwinds the RNA duplex, resulting in a complex composed of DDX3, single-stranded RNA (ssRNA), and ATP. ATP hydrolysis then facilitates the release of ssRNA [43,44]. The capsid protein interaction of domain 2 of DDX3 might affect its RNAbinding.

The HEV capsid protein contains three major domains, S, M, and P [45]. After synthesis, the capsid protein forms dimers and further assemblies into icosahedral virus-like particles (VLP). The crystal structure of the VLP shows that all of the three domains have some area exposed to the surface of the VLP, with the P domain protruding outside [45]. A total of 30 dimeric spikes are situated on the icosahedral 2-fold symmetry axes. Our computational model predicted that DDX3 and the capsid protein interact via $\mathrm{C}$-terminal domains. The co-IP result showed that the C-terminal domain of DDX3 is the predominant interaction site. This interaction is not required for HEV replication as the K230 ATPase motif at the N-terminal domain of DDX3 is indispensable for viral proliferation. These results indicate that DDX3 plays a pro-viral role in HEV replication, while its assistance of IFN induction is blocked by the capsid protein. Further research is warranted to elucidate the mechanism of the DDX3 involvement in HEV infection.

DDX3 is known to be required for some viral replication [12]. During HCV replication, the DDX3 C-terminal domain interacts with the N-terminal 59 aa of $\mathrm{HCV}$ core protein independent of RNA [46]. Moreover, DDX3 is recruited to the lipid droplets, where HCV virions assemble, and knockdown of DDX3 in hepatoma cells significantly inhibits HCV replication [13]. A later study shows that a single amino acid mutation within the N-terminal domain of HCV core protein abrogates its interaction with DDX3 without affecting the virus propagation. After the mutation of the core protein gene in the virus genome, DDX3 does not co-localize with the core protein in HCV-replicating hepatoma cells anymore. However, the silencing of DDX3 still results in inhibition of the HCV replication, indicating that the interaction between DDX3 and the core protein is not required for the viral replication, and the pro-viral role of DDX3 is independent of its subcellular translocation [47]. Another report demonstrated that DDX3 augments MAVS-mediated increase in IFN production through direct interaction with MAVS [11], while this promotion can be counteracted by the HCV core protein, possibly due to the hijack of DDX3 by the core protein from RIG-I/MAVS/DDX3 complex to lipid droplets [11]. DDX3 also contributes to the viral replication of the Japanese encephalitis virus (JEV). The absence of DDX3 leads to defective viral replication without affecting viral assembly and release. Overexpression of the DDX3 with an RNA helicase motif mutation, S382L, does not rescue viral replication, indicating a critical role of the RNA helicase activity of DDX3. 
Additionally, DDX3 co-localizes with viral NS3, NS5, and dsRNA, and interacts with 5'UTR and 3'UTR to facilitate viral replication [48].

In conclusion, the HEV capsid protein interacts with DDX3, and DDX3 is indispensable for HEV replication. The ATPase activity of DDX3 is involved in the HEV replication. These results reveal HEV regulation of DDX3 for a conducive environment of replication and provide further insights into the virus-cell interactions.

Author Contributions: S.L. and Y.Z. contributed to the study design. S.L., P.C., J.H., E.C., B.G.P., and Y.Z. were involved in data acquisition, analysis, and drafted the manuscript. All authors reviewed the manuscript.

Funding: This work was supported by a seed grant from the VA-MD College of Veterinary Medicine, the University of Maryland to Y.Z.

Acknowledgment: We thank Dr. Suzanne Emerson at NIH for HEV Kernow-C1 p6 and p6/luc, and chimpanzee antibody against the HEV capsid protein.

Conflicts of Interests: The authors declare no conflicts of interest.

\section{References}

1. Singleton, M.R.; Dillingham, M.S.; Wigley, D.B. Structure and mechanism of helicases and nucleic acid translocases. Annu Rev Biochem 2007, 76, 23-50, doi:10.1146/annurev.biochem.76.052305.115300.

2. Cordin, O.; Banroques, J.; Tanner, N.K.; Linder, P. The DEAD-box protein family of RNA helicases. Gene 2006, 367, 17-37, doi:10.1016/j.gene.2005.10.019.

3. Riva, V.; Maga, G. From the magic bullet to the magic target: exploiting the diverse roles of DDX3X in viral infections and tumorigenesis. Future Med Chem 2019, 11, 1357-1381, doi:10.4155/fmc-2018-0451.

4. Schroder, M.; Baran, M.; Bowie, A.G. Viral targeting of DEAD box protein 3 reveals its role in TBK1/IKKepsilon-mediated IRF activation. The EMBO journal 2008, 27, 2147 2157, doi:10.1038/emboj.2008.143.

5. Szappanos, D.; Tschismarov, R.; Perlot, T.; Westermayer, S.; Fischer, K.; Platanitis, E.; Kallinger, F.; Novatchkova, M.; Lassnig, C.; Muller, M., et al. The RNA helicase DDX $3 \mathrm{X}$ is an essential mediator of innate antimicrobial immunity. PLoS Pathog 2018, 14, e1007397, doi:10.1371/journal.ppat.1007397.

6. Chan, C.H.; Chen, C.M.; Lee, Y.W.; You, L.R. DNA Damage, Liver Injury, and Tumorigenesis: Consequences of DDX3X Loss. Mol Cancer Res 2019, 17, 555-566, doi:10.1158/1541-7786.MCR-18-0551.

7. Matsumura, T.; Endo, T.; Isotani, A.; Ogawa, M.; Ikawa, M. An azoospermic factor gene, Ddx3y and its paralog, Ddx3x are dispensable in germ cells for male fertility. $J$ Reprod Dev 2019, 65, 121-128, doi:10.1262/jrd.2018-145.

8. Kim, Y.S.; Lee, S.G.; Park, S.H.; Song, K. Gene structure of the human DDX3 and chromosome mapping of its related sequences. Mol Cells 2001, 12, 209-214.

9. Foresta, C.; Ferlin, A.; Moro, E. Deletion and expression analysis of AZFa genes on the human $\mathrm{Y}$ chromosome revealed a major role for DBY in male infertility. Human Molecular Genetics 2000, 9, 1161-1169, doi:10.1093/hmg/9.8.1161. 
10. Yedavalli, V.S.; Neuveut, C.; Chi, Y.H.; Kleiman, L.; Jeang, K.T. Requirement of DDX3 DEAD box RNA helicase for HIV-1 Rev-RRE export function. Cell 2004, 119, 381-392, doi:10.1016/j.cell.2004.09.029.

11. Oshiumi, H.; Sakai, K.; Matsumoto, M.; Seya, T. DEAD/H BOX 3 (DDX3) helicase binds the RIG-I adaptor IPS-1 to up-regulate IFN-beta-inducing potential. Eur J Immunol 2010, 40, 940-948, doi:10.1002/eji.200940203.

12. Taschuk, F.; Cherry, S. DEAD-Box Helicases: Sensors, Regulators, and Effectors for Antiviral Defense. Viruses 2020, 12, doi:10.3390/v12020181.

13. Ariumi, Y.; Kuroki, M.; Abe, K.; Dansako, H.; Ikeda, M.; Wakita, T.; Kato, N. DDX3 DEAD-box RNA helicase is required for hepatitis C virus RNA replication. $J$ Virol 2007, 81, 13922-13926, doi:10.1128/JVI.01517-07.

14. Loureiro, M.E.; Zorzetto-Fernandes, A.L.; Radoshitzky, S.; Chi, X.; Dallari, S.; Marooki, N.; Leger, P.; Foscaldi, S.; Harjono, V.; Sharma, S., et al. DDX3 suppresses type I interferons and favors viral replication during Arenavirus infection. PLoS Pathog 2018, 14, e1007125, doi:10.1371/journal.ppat.1007125.

15. Thulasi Raman, S.N.; Liu, G.; Pyo, H.M.; Cui, Y.C.; Xu, F.; Ayalew, L.E.; Tikoo, S.K.; Zhou, Y. DDX3 Interacts with Influenza A Virus NS1 and NP Proteins and Exerts Antiviral Function through Regulation of Stress Granule Formation. J Virol 2016, 90, 3661-3675, doi:10.1128/JVI.03010-15.

16. Wang, H.; Kim, S.; Ryu, W.S. DDX3 DEAD-Box RNA helicase inhibits hepatitis B virus reverse transcription by incorporation into nucleocapsids. J Virol 2009, 83, 5815-5824, doi:10.1128/JVI.00011-09.

17. Jameel, S. Molecular biology and pathogenesis of hepatitis E virus. Expert Rev Mol Med 1999, 1999, 1-16, doi:10.1017/S1462399499001271.

18. $\quad$ Purcell, R.H.; Emerson, S.U. Hepatitis E virus. In Fields Virology, Fourth Edition ed.; Knipe, D.M., Howley, P.M., Eds. Lippincott Williams \& Wilkins: Philadelphia, 2001; Vol. 2, pp. 3051-3061.

19. Perez-Gracia, M.T.; Suay-Garcia, B.; Mateos-Lindemann, M.L. Hepatitis E and pregnancy: current state. Rev Med Virol 2017, 27, e1929, doi:10.1002/rmv.1929.

20. Lachish, T.; Erez, O.; Daudi, N.; Shouval, D.; Schwartz, E. Acute hepatitis E virus in pregnant women in Israel and in other industrialized countries. J Clin Virol 2015, 73, 20 24, doi:10.1016/j.jcv.2015.10.011.

21. Lin, S.; Yang, Y.; Nan, Y.; Ma, Z.; Yang, L.; Zhang, Y.J. The Capsid Protein of Hepatitis E Virus Inhibits Interferon Induction via Its N-terminal Arginine-Rich Motif. Viruses 2019, 11, 1050, doi:10.3390/v11111050.

22. Zhong, J.; Gastaminza, P.; Cheng, G.; Kapadia, S.; Kato, T.; Burton, D.R.; Wieland, S.F.; Uprichard, S.L.; Wakita, T.; Chisari, F.V. Robust hepatitis C virus infection in vitro. Proc Natl Acad Sci U S A 2005, 102, 9294-9299, doi:10.1073/pnas.0503596102.

23. Shukla, P.; Nguyen, H.T.; Faulk, K.; Mather, K.; Torian, U.; Engle, R.E.; Emerson, S.U. Adaptation of a genotype 3 hepatitis E virus to efficient growth in cell culture depends on an inserted human gene segment acquired by recombination. Journal of virology 2012, 86, 5697-5707, doi:10.1128/JVI.00146-12.

24. Kim, D.I.; Jensen, S.C.; Noble, K.A.; Kc, B.; Roux, K.H.; Motamedchaboki, K.; Roux, K.J. An improved smaller biotin ligase for BioID proximity labeling. Molecular biology of the cell 2016, 27, 1188-1196, doi:10.1091/mbc.E15-12-0844. 
25. Roux, K.J.; Kim, D.I.; Burke, B.; May, D.G. BioID: A Screen for Protein-Protein Interactions. Current protocols in protein science 2018, 91, 19.23.11-19.23.15, doi:10.1002/cpps.51.

26. Shevchenko, A.; Wilm, M.; Vorm, O.; Mann, M. Mass spectrometric sequencing of proteins silver-stained polyacrylamide gels. Analytical chemistry 1996, 68, 850-858, doi:10.1021/ac950914h.

27. Eng, J.K.; McCormack, A.L.; Yates, J.R. An approach to correlate tandem mass spectral data of peptides with amino acid sequences in a protein database. J Am Soc Mass Spectrom 1994, 5, 976-989, doi:10.1016/1044-0305(94)80016-2.

28. Mellacheruvu, D.; Wright, Z.; Couzens, A.L.; Lambert, J.P.; St-Denis, N.A.; Li, T.; Miteva, Y.V.; Hauri, S.; Sardiu, M.E.; Low, T.Y., et al. The CRAPome: a contaminant repository for affinity purification-mass spectrometry data. Nat Methods 2013, 10, 730736, doi:10.1038/nmeth.2557.

29. Nan, Y.; Yu, Y.; Ma, Z.; Khattar, S.K.; Fredericksen, B.; Zhang, Y.J. Hepatitis E virus inhibits type I interferon induction by ORF1 products. J Virol 2014, 88, 11924-11932, doi:10.1128/JVI.01935-14.

30. Nan, Y.; Wang, R.; Shen, M.; Faaberg, K.S.; Samal, S.K.; Zhang, Y.J. Induction of type I interferons by a novel porcine reproductive and respiratory syndrome virus isolate. Virology 2012, 432, 261-270, doi:10.1016/j.virol.2012.05.015.

31. Yamashita, T.; Mori, Y.; Miyazaki, N.; Cheng, R.H.; Yoshimura, M.; Unno, H.; Shima, R.; Moriishi, K.; Tsukihara, T.; Li, T.C., et al. Biological and immunological characteristics of hepatitis E virus-like particles based on the crystal structure. Proc Natl Acad Sci U S A 2009, 106, 12986-12991, doi:10.1073/pnas.0903699106.

32. Rose, P.W.; Beran, B.; Bi, C.; Bluhm, W.F.; Dimitropoulos, D.; Goodsell, D.S.; Prlic, A.; Quesada, M.; Quinn, G.B.; Westbrook, J.D., et al. The RCSB Protein Data Bank: redesigned web site and web services. Nucleic Acids Res 2011, 39, D392-401, doi:10.1093/nar/gkq1021.

33. Floor, S.N.; Condon, K.J.; Sharma, D.; Jankowsky, E.; Doudna, J.A. Autoinhibitory Interdomain Interactions and Subfamily-specific Extensions Redefine the Catalytic Core of the Human DEAD-box Protein DDX3. J Biol Chem 2016, 291, 2412-2421, doi:10.1074/jbc.M115.700625.

34. Pierce, B.G.; Wiehe, K.; Hwang, H.; Kim, B.H.; Vreven, T.; Weng, Z. ZDOCK server: interactive docking prediction of protein-protein complexes and symmetric multimers. Bioinformatics 2014, 30, 1771-1773, doi:10.1093/bioinformatics/btu097.

35. Lin, S.; Yang, Y.; Nan, Y.; Ma, Z.; Yang, L.; Zhang, Y. The Capsid Protein of Hepatitis E Virus Inhibits Interferon Induction via Its N-terminal Arginine-Rich Motif. Viruses 2019, 11, 1050 .

36. Kang, J.I.; Kwon, Y.C.; Ahn, B.Y. Modulation of the type I interferon pathways by culture-adaptive hepatitis C virus core mutants. FEBS Lett 2012, 586, 1272-1278, doi:10.1016/j.febslet.2012.03.062.

37. Graff, J.; Torian, U.; Nguyen, H.; Emerson, S.U. A bicistronic subgenomic mRNA encodes both the ORF2 and ORF3 proteins of hepatitis E virus. J Virol 2006, 80, 59195926, doi:10.1128/JVI.00046-06.

38. Shukla, P.; Nguyen, H.T.; Faulk, K.; Mather, K.; Torian, U.; Engle, R.E.; Emerson, S.U. Adaptation of a genotype 3 hepatitis E virus to efficient growth in cell culture depends on 
an inserted human gene segment acquired by recombination. $J$ Virol 2012, 86, 56975707, doi:10.1128/JVI.00146-12.

39. Soto-Rifo, R.; Rubilar, P.S.; Limousin, T.; de Breyne, S.; Decimo, D.; Ohlmann, T. DEAD-box protein DDX3 associates with eIF4F to promote translation of selected mRNAs. The EMBO journal 2012, 31, 3745-3756, doi:10.1038/emboj.2012.220.

40. Geissler, R.; Golbik, R.P.; Behrens, S.E. The DEAD-box helicase DDX3 supports the assembly of functional 80S ribosomes. Nucleic Acids Res 2012, 40, 4998-5011, doi:10.1093/nar/gks070.

41. Hogbom, M.; Collins, R.; van den Berg, S.; Jenvert, R.M.; Karlberg, T.; Kotenyova, T.; Flores, A.; Karlsson Hedestam, G.B.; Schiavone, L.H. Crystal structure of conserved domains 1 and 2 of the human DEAD-box helicase DDX3X in complex with the mononucleotide AMP. J Mol Biol 2007, 372, 150-159, doi:10.1016/j.jmb.2007.06.050.

42. $\quad$ Epling, L.B.; Grace, C.R.; Lowe, B.R.; Partridge, J.F.; Enemark, E.J. Cancer-associated mutants of RNA helicase DDX3X are defective in RNA-stimulated ATP hydrolysis. Journal of molecular biology 2015, 427, 1779-1796, doi:10.1016/j.jmb.2015.02.015.

43. Liu, F.; Putnam, A.; Jankowsky, E. ATP hydrolysis is required for DEAD-box protein recycling but not for duplex unwinding. Proceedings of the National Academy of Sciences of the United States of America 2008, 105, 20209-20214, doi:10.1073/pnas.0811115106.

44. Chen, Y.; Potratz, J.P.; Tijerina, P.; Del Campo, M.; Lambowitz, A.M.; Russell, R. DEAD-box proteins can completely separate an RNA duplex using a single ATP. Proceedings of the National Academy of Sciences of the United States of America 2008, 105, 20203-20208, doi:10.1073/pnas.0811075106.

45. Guu, T.S.; Liu, Z.; Ye, Q.; Mata, D.A.; Li, K.; Yin, C.; Zhang, J.; Tao, Y.J. Structure of the hepatitis E virus-like particle suggests mechanisms for virus assembly and receptor binding. Proc Natl Acad Sci U S A 2009, 106, 12992-12997, doi:10.1073/pnas.0904848106.

46. Owsianka, A.M.; Patel, A.H. Hepatitis C virus core protein interacts with a human DEAD box protein DDX3. Virology 1999, 257, 330-340, doi:10.1006/viro.1999.9659.

47. Angus, A.G.; Dalrymple, D.; Boulant, S.; McGivern, D.R.; Clayton, R.F.; Scott, M.J.; Adair, R.; Graham, S.; Owsianka, A.M.; Targett-Adams, P., et al. Requirement of cellular DDX3 for hepatitis $\mathrm{C}$ virus replication is unrelated to its interaction with the viral core protein. J Gen Virol 2010, 91, 122-132, doi:10.1099/vir.0.015909-0.

48. Li, C.; Ge, L.L.; Li, P.P.; Wang, Y.; Dai, J.J.; Sun, M.X.; Huang, L.; Shen, Z.Q.; Hu, X.C.; Ishag, H., et al. Cellular DDX3 regulates Japanese encephalitis virus replication by interacting with viral un-translated regions. Virology 2014, 449, 70-81, doi:10.1016/j.virol.2013.11.008. 